Ciencia y Educación, Vol. 5, No. 2, mayo-agosto, 2021

ISSN (impreso): 2613-8794・ISSN (en línea): 2613-8808

DOI: https://doi.org/10.22206/cyed.2021.v5i2.pp25-40

\title{
Revisión actualizada: enseñanza de las matemáticas desde los entornos virtuales de aprendizaje
}

\author{
Updated review: Mathematics teaching from virtual \\ learning environments
}

Jasmín Corrales Jaar ${ }^{a}$ ORCID: 0000-0002-9769-9780

Recibido: 18/06/2020 • Aprobado: 27/10/2020

Cómo citar: Corrales Jaar, J. (2021). Revisión actualizada: enseñanza de las matemáticas desde los entornos virtuales de aprendizaje. Ciencia y Educación, 5(2), 25-40. https://doi.org/10.22206/cyed.2021.v5i2.pp25-40

\section{Resumen}

La necesidad de implementar los entornos virtuales de aprendizaje (EVA) es hoy, a raíz de la inédita situación generada por la pandemia del Covid19 y el trabajo escolar desde la no presencialidad, claramente justificada, dado que es una forma plausible de desarrollar las usuales actividades escolares. Esta investigación de carácter interpretativo-cualitativo tuvo como objetivo desarrollar una revisión de las investigaciones realizadas sobre los entornos virtuales de aprendizaje implementados para promover los procesos enseñanza y aprendizaje de las matemáticas. Para esto, se consultó en las bases de datos ERIC y Scielo. Se utilizaron descriptores y, posteriormente, mediante procesos de preselección, cribado y selección, se incluyeron 19 artículos para su estudio. Los hallazgos obtenidos muestran que la mayoría de ellos apunta sobre la eficacia de los EVA y las TIC para facilitar aprendizajes significativos de los contenidos matemáticos, aumentar la motivación y la metacognición.

Palabras clave: Entornos Virtuales de Aprendizaje, TIC, enseñanza de las matemáticas, tecnología educativa, docentes.

\begin{abstract}
The need to implement virtual learning environments (VLE) is today, as a result of the unprecedented situation generated by the Covid 19 pandemic and schoolwork from non-presence, clearly justified, since it is a plausible way to develop the usual school activities. This interpretive-qualitative research aimed to develop a review of the research carried out on virtual learning environments implemented to promote the teaching and learning processes of Mathematics. For this, the ERIC and Scielo databases were consulted. Descriptors were used and later, through pre-selection, screening and selection processes, 19 articles were finally included for study. The findings obtained show that most of them point to the effectiveness of VAS and ICT to facilitate meaningful learning of mathematical content, increase motivation and metacognition.
\end{abstract}

Keywords: Virtual learning environments, ICT, mathematical teaching, Educative technology, teachers.

\footnotetext{
a Universidad Pedagógica Experimental Libertador (UPEL), Venezuela. Correo: corralesjaarjasmin@sanjosedistrital.edu.co
} 


\section{Introducción}

La tecnología es un componente esencial y ubicuo de nuestra sociedad. La actual crisis generada por el Covid19 ha demostrado que gracias a la tecnología $\mathrm{y}$ a las herramientas digitales podemos acceder de alguna forma a nuestro trabajo, a lugares distantes, a la información y a procesos de enseñanza y aprendizaje. Aunque hay diversas fuentes que registran los impactos positivos de las Tecnologías de la Información y las Comunicaciones [TIC] sobre los estudiantes, el uso e integración a la enseñanza por parte del profesorado ha sido lento (Díaz, de Luna y Salinas, 2019; Hollebrands, 2017).

De la misma manera, las matemáticas son consideradas como fundamentales para los estudiantes de todo el mundo, pues se incluyen como parte de su formación y les permitirá mejorar su calidad de vida (Siaw, Gayabaah y Boeteng, 2016; Organización de las Naciones Unidas para la Educación, la Ciencia y la Cultura-Laboratorio Latinoamericano para la Educación en América Latina y el Caribe [UNESCO/ LLECE], 2015; 2016).

No obstante, los bajos rendimientos académicos son un problema generalizado al que se enfrenta el profesorado de matemáticas en todos los niveles. Así lo registran autores como Díaz, de Luna y Salinas (2019); Gómez (2011); González (2013); y Petri, Barona, López y Quiroz (2010). A lo anterior, se suma los bajos índices de motivación y las actitudes negativas hacia el aprendizaje de los contenidos matemáticos por parte de los estudiantes. Además, el profesorado continúa aplicando desde su praxis pedagógica métodos tradicionales en los cuales se espera que los estudiantes sean simples receptores de clases y que lean el libro de texto sugerido. Como consecuencia, se privilegia la memorización aislada de los conceptos matemáticos y de lecciones sin comprensión, lo cual empeora la situación descrita (Siaw, Gayabaah y Boeteng, 2016; Stošic, 2015).

Sin embargo, existe la posibilidad de integrar el uso de las TIC al ámbito educativo con el propósito de generar transformaciones en todos los niveles, desde la adaptación de los currículos y las formas tradicionales de enseñar e interactuar, hasta el diseño e implementación de estrategias y propuestas didácticas, con miras a responder satisfactoriamente a los desafíos de la sociedad.

En este sentido, los docentes como parte vertebral de los procesos de enseńanza y aprendizaje deben estar dispuestos a adoptar nuevas formas de enseñar, de modo que promuevan aprendizajes significativos, interactivos y que estimulen la motivación de los estudiantes mediante el uso de las TIC, el uso de software, herramientas móviles y la creación de entornos virtuales de aprendizaje [EVA] (Olivo-Franco y Corrales, 2020; Díaz, Lagunes, López y Recio, 2012).

Es necesario abordar algunos constructos importantes para considerar el asunto de las TIC, los EVA, así como las herramientas electrónicas y digitales aplicadas en el campo educativo, en particular, de la enseñanza de las matemáticas, como son: el Constructivismo y la Teoría del Conectivismo.

En el caso del Constructivismo, este constituye un modelo epistemológico que se opone al objetivismo del empirismo, en contraste con el relativismo del conocimiento y la noción de una realidad construida (Martínez, 2012; Vélez y Galeano, 2002). En esa misma línea, Seitzinger (2006) declara expresamente que el aprendizaje en línea se apoya en una pedagogía constructivista y posteriormente destaca algunas características que deben imperar en este tipo de aprendizaje, tales como: ser activo y manipulable, ser constructivo y reflexivo, ser intencional, ser auténtico, ser cooperativo, colaborativo y conversacional.

Ahora bien, la teoría general de los Sistemas, la Teoría Cibernética y la Teoría de la Información emergidas en el Constructivismo abrieron toda una perspectiva a través de sustentar el cambio de las formas tradicionales de desarrollar los procesos de enseńanza y aprendizaje (Osorio, 2012), porque tal como lo señalan Olivo-Franco y Corrales (2020) posibilitan:

Capacidad de comprenderse de una manera distinta la que ofrece posibilidades de resignificar la praxis docente en este caso de las Matemáticas, desde la transdisciplinariedad, lo cual implica modificar los criterios tradicionales de seleccionar los contenidos a tratar, las formas de relacionarse con los estudiantes, en fin, las formas de concebir 
la realidad educativa. Estos, sin duda son pasos necesarios en la implementación de entornos virtuales de aprendizaje. (p.11)

Desde la Teoría del Conectivismo, el aprendizaje puede verificarse de diversas formas, como por ejemplo: "a través de comunidades prácticas, redes personales y mediante la realización de tareas relacionadas al trabajo" (Simiens, 2004, p. 1). De hecho, Bertanlanffy (1974) había propuesto pensar en términos de conectividades, relaciones y contextos. Por su parte, Driscoll (2000) agrega que desde el conectivismo el aprendizaje se efectúa por la construcción de vínculos entre los elementos de la información, en tanto que este puede residir en los dispositivos no humanos.
El conectivismo supone la idea de que se propicia el aprendizaje de los individuos al promover la creación de redes institucionales formales y no formales que incorporen la tecnología y las redes para, a su vez, facilitar acceder a nuevos saberes y conocimientos generados por la sociedad (Solórzano y García, 2016).

$\mathrm{Al}$ respecto, Olivo-Franco y Corrales (2020) ofrecen una excelente síntesis de los principales sustentos teóricos del Conectivismo, así como algunos principios rectores para la implementación del aprendizaje en redes en el aula sugeridos desde los principios clave del constructivismo y conectivismo según Solórzano y García (2016), que se resumen en las Tablas 1 y 2.

\section{Tabla 1}

Principales sustentos teóricos del Conectivismo

\begin{tabular}{|c|c|c|}
\hline $\begin{array}{l}\text { Teóricas que permiten su } \\
\text { fundamentación }\end{array}$ & Aporte & Principios del Conectivismo (Siemens, 2004) \\
\hline $\begin{array}{l}\text { Teoría sociohistórica } \\
\text { de Vygotsky }\end{array}$ & $\begin{array}{c}\text { El aprendizaje resulta de la interacción entre } \\
\text { sujetos y medio, lo cual incluye } \\
\text { la información. }\end{array}$ & \\
\hline $\begin{array}{l}\text { Teoría general de sistemas } \\
\text { (Bertalanffy, 1974). }\end{array}$ & $\begin{array}{l}\text { Propone el pensamiento sistémico y este, } \\
\text { a su vez, propone pensar en términos de } \\
\text { conectividades, relaciones y contextos. }\end{array}$ & $\begin{array}{c}\text {-Aprendizaje y conocimiento ubicados en la } \\
\text { diversidad de opiniones. }\end{array}$ \\
\hline $\begin{array}{c}\text { Teoría cibernética (Wiener, según } \\
\text { Moreno, 2003) }\end{array}$ & $\begin{array}{c}\text { Entendida como el campo interdisciplinario } \\
\text { que aborda los problemas de la organización } \\
\text { y los procesos de control (retroalimentación) } \\
\text { y transmisión de informaciones } \\
\text { (comunicación), en las máquinas } \\
\text { y los organismos vivos. }\end{array}$ & $\begin{array}{c}\text { El aprendizaje como proceso de conexión } \\
\text { especializada de nodos o fuentes de información. } \\
\text {-El aprendizaje puede residir en artefactos no } \\
\text { humanos. }\end{array}$ \\
\hline $\begin{array}{l}\text { Teoría de la información Shannon, } \\
\text { Weaver, Foerster, Jakobson, Winkin, } \\
\text { Bateson, Ruesh, Birdwhistell, } \\
\text { Scheflen, Hall y Gofman (según } \\
\text { Moreno, 2003) }\end{array}$ & $\begin{array}{l}\text { La comunicación es definida como un } \\
\text { proceso social que integra múltiples modos } \\
\text { de comportamiento, considerada como un } \\
\text { todo integrado, regido por un conjunto } \\
\text { de reglas y códigos determinados por cada } \\
\text { cultura. Con los aportes de la cibernética } \\
\text { y la sistémica. }\end{array}$ & $\begin{array}{l}\text { actualmente conocido. } \\
\text { Alimentar y mantener las conexiones es vital para un } \\
\text { aprendizaje continuo. } \\
\text { Es esencial la habilidad para identificar conexiones } \\
\text { entre áreas, ideas y conceptos. } \\
\text {-La toma de decisiones es un aprendizaje en sí mismo. }\end{array}$ \\
\hline $\begin{array}{l}\text { Pensamiento complejo (Maturana } \\
\text { y Varela, 1990; Morin, 1996, entre } \\
\text { otros) }\end{array}$ & $\begin{array}{l}\text { Propone la apertura hacia el pensamiento } \\
\text { complejo en vista de la crisis del } \\
\text { pensamiento de la simplificación y } \\
\text { reduccionismo de la ciencia tradicional. En } \\
\text { contraste, invita a pensar de manera integral, } \\
\text { transversal, transdisciplinar, lo cual, sin } \\
\text { duda, es coherente con la ideas } \\
\text { del Conectivismo. }\end{array}$ & $\begin{array}{c}\text {-Seleccionar qué aprender y el significado de la } \\
\text { información entrante es visto a través de los lentes de } \\
\text { una realidad cambiante. }\end{array}$ \\
\hline
\end{tabular}

Nota. Tomado de Olivo-Franco y Corrales (2020). 
Tabla 2

Algunos principios rectores para la implementación de redes de aprendizaje en el aula según Solórzano y García (2016)

\begin{tabular}{|c|c|c|}
\hline $\begin{array}{c}\text { Principios para el diseńo de una propuesta } \\
\text { de aprendizaje en red desde el conectivismo } \\
\text { y la Teoría } \\
\text { de la Actividad }\end{array}$ & $\begin{array}{c}\text { Ideas rectoras para el diseńo de una } \\
\text { propuesta de aprendizaje en red desde el } \\
\text { Conectivismo y la Teoría de } \\
\text { la Actividad }\end{array}$ & $\begin{array}{c}\text { Guía para Propuesta de aprendizaje } \\
\text { en red en el aula }\end{array}$ \\
\hline
\end{tabular}

El conocimiento conectivo es la base del aprendizaje en red.

-El conocimiento por intermedio de las interconexiones puede residir en el colectivo.

-La capacidad de establecer distinciones entre la información importante y sin importancia es vital.

-El carácter social de aprendizaje expansivo en redes.

-Carácter activo del estudiante como sujeto en red.

-La integración y cohesión de un grupo es producto del desarrollo de tareas y objetivos comunes.

-Relación actividad tecnológica digitalconexiones.
La institución debe facilitar el acceso de los actores a la tecnología y brindar capacitación permanente.

-Cada actor debe construir su propio entorno personal de aprendizaje (EPA) y luego construir redes de aprendizaje que satisfaga intereses comunes.

-Los docentes deben facilitar la creación de redes de aprendizaje y promover espacios virtuales con la construcción de redes de aprendizaje específicas que permitan a los estudiantes expresarse.

-Debe existir libertad de acceder al conocimiento a través de las redes de aprendizaje.
1. Diagnóstico del aprendizaje digital en red, de las herramientas con que cuenta la institución educativa y el dominio que tienen los estudiantes de estas herramientas.

2. Capacitación en el uso de herramientas de la Web 2.0, Web 3.0 y herramientas que servirán para relacionarse con otros mediante redes de aprendizaje, redes temáticas o sociales.

3. Construcción de un EPA.

4. Diseño y orientación de actividades de aprendizaje.

5. Creación de una red de aprendizaje y dinamizar la misma red.

6. Desarrollo de las actividades y elaboración de productos.

7. Retroalimentación en los procesos de elaboración, implementación y evaluación final.

8. Evaluación

Nota. Tomado de Olivo-Franco y Corrales (2020).

\section{Metodología}

El paradigma interpretativo abre múltiples posibilidades para comprender la complejidad del entorno social (Hernández, Fernández y Baptista, 2010; Martínez, 2012). Dentro de este marco epistemológico la revisión bibliográfica es una investigación que recoge, analiza y sintetiza la información más importante sobre un tópico determinado (Rubio y OlivoFranco, 2020a).

Ahora bien, aplicar este método significó seguir con flexibilidad las siguientes etapas: 1) establecer con claridad los objetivos de la investigación; 2) ejecución de la búsqueda bibliográfica, es decir, consultar las bases de datos seleccionadas y establecer criterios de selección, inclusión y exclusión de los documentos;
3) organizar la información y 4) analizar, sintetizar y exponer la información destacada sobre el objeto o fenómeno estudiado (Day, 2005; Guirao-Goris, Olmedo y Ferrer, 2008).

Otras características importantes relacionadas con la revisión bibliográfica como investigación son: 1) es reconstructiva, pues permite rehacer las rutas y los caminos que han seguido los investigadores para desarrollar sus investigaciones; 2) descriptiva, conceptual y sistemática, debido a que permite estructurar formalmente la idea primigenia de investigación, siguiendo un proceso organizado de indagación, recolección, organización de información sobre un tópico particular (Morales, 2015, Orozco-Alvarado y Díaz-Pérez, 2018); 3) hermenéutica, en tanto que posibilita la interpretación del fenómeno estudiado; de construcción 
interdisciplinaria y 4) continua, puesto que permite generar ideas sobre lo investigado, plantear concepciones propias y, en resumen, tal como lo señala Morales (2015), le permite al investigador "ser un creador" (p. 217). En este sentido, autores como Hernández, Fernández y Baptista (2010) y Vargas (1988) coinciden con planteamientos similares a los expuestos anteriormente sobre la investigación documental.

Este trabajo plantea realizar una revisión de investigaciones desarrolladas durante el año 2016 hasta el primer trimestre de 2020 sobre las TIC y los EVA en la enseñanza de las matemáticas. Deben hacer parte del repositorio de las bases de datos ERIC y Scielo. Asimismo, a partir de la información recabada ofrecer sugerencias y aportes que puedan contribuir a mejorar la praxis y el quehacer pedagógico del profesorado de la asignatura en cuestión, porque hacerlo supone aproximarse a fomentar mejores aprendizajes. Esto último si se plantea desde una concepción dialógica de concepción de los procesos de enseñanza y aprendizaje.

Por tanto, el estudio verificó artículos que enmarcan propuestas con relación al profesorado, como aquellos en los cuales los autores fueron los mismos docentes, quienes implementaron, diseñaron y evaluaron estrategias virtuales, aplicación de las TIC y herramientas digitales de aprendizaje en el contexto de la enseńanza y el aprendizaje de la matemática. Se desarrolló así una revisión bibliográfica tendiendo a los criterios de selección registrados en la Tabla 3 y guiados por los siguientes objetivos de investigación:

Revisar las investigaciones sobre entornos virtuales y aplicaciones TIC en la enseñanza o instrucción de las matemáticas registrados en las bases de datos ERIC y Scielo desde 2016 hasta el primer trimestre de 2020.

- Analizar los principales aportes ofrecidos por tales estudios en la promoción de una nueva praxis de la enseñanza de las matemáticas mediada por los entornos virtuales de aprendizaje.

- Aportar al profesorado de matemáticas algunas recomendaciones que faciliten la praxis de la enseñanza de las matemáticas mediadas por las TIC, hacia la promoción de aprendizajes significativos en los estudiantes.

\section{Tabla 3}

Criterios de inclusión y selección

\begin{tabular}{cc}
\hline Criterios & Descripción del criterio \\
\hline Periodo & $2016-2020$ \\
\hline Formato & $\begin{array}{c}\text { Artículo de revista científica revisada } \\
\text { por pares }\end{array}$ \\
\hline Disciplinas & Educación y Psicología \\
\hline Idiomas & Inglés, español \\
\hline $\begin{array}{l}\text { Otros criterios } \\
\text { de inclusión o } \\
\text { limitadores }\end{array}$ & $\begin{array}{c}\text { Instrucción matemática, tecnología } \\
\text { educativa, métodos de enseñanza. }\end{array}$ \\
\hline
\end{tabular}

TitleCombined:(“entornos virtuales de aprendizaje”) AND (“matemáticas”))

Ecuación de búsqueda

OR

TitleCombined:("teach" AND "match" AND "e-learning" AND "virtuality)

\section{Resultados}

La búsqueda arrojó un total de 61 artículos académicos (59 en ERIC y 2 en Scielo), de los cuales se seleccionaron 19 por ser los que cumplieron con los criterios de inclusión ya señalados. Se evidencia que los artículos de investigación analizados abordan los campos de la enseñanza o instrucción matemática en el nivel de educación primaria o secundaria, universitaria $y$, en su gran mayoría, apuntan hacia la formación del profesorado de matemáticas. En cuanto al contexto en el cual se desarrollaron, destacan países como Arabia Saudita, Australia, Corea, Estados Unidos, Hong Kong, Ghana, Noruega, Turquía, República Checa, Rusia y México.

Los estudios revisados sobre enseñanza de las matemáticas, mediada por entornos de aprendizaje virtual o por estrategias que involucran aprendizaje electrónico, medios digitales y TIC, demuestran ser eficaces. Lo anterior se evidencia en la medida en que promueven el desarrollo de habilidades matemáticas, la internalización de conceptos matemáticos, geométricos o algebraicos. En el caso del profesorado, este declara que la implementación de los EVA puede incidir en las prácticas pedagógicas, de forma que esta sea transversal. 
Con miras a responder el primer y segundo objetivo planteado en el presente estudio se desarrolló la Tabla 4, que permite verificar algunas características de los artículos analizados, así como un resumen de los principales aportes a la enseñanza y al aprendizaje de las matemáticas.

\section{Tabla 4}

Principales caracteristicas de los estudios sobre enseñanza de las matemáticas a partir de EVA, TIC o mediados por herramientas digitales

\begin{tabular}{|c|c|c|c|}
\hline Autor & Contexto & Título & Principales aportes \\
\hline $\begin{array}{c}\text { Bozkurt } \\
(2016)\end{array}$ & Turquía & $\begin{array}{l}\text { Profesores de matemáticas } \\
\text { y TIC: factores que afectan } \\
\text { el uso previo al servicio en } \\
\text { las prácticas escolares }\end{array}$ & $\begin{array}{l}\text { Señala que las barreras del uso de las TIC por los futuros profesores } \\
\text { parecen relacionarse con: } \\
\text { acceso deficiente a las instalaciones de las TIC, estímulo limitado } \\
\text { por parte de los mentores en matemáticas y uso irregular de las TIC } \\
\text { por parte de los profesores existentes en la docencia, falta de vínculos } \\
\text { en el esquema de trabajo y limitación de tiempo. El desafío para los } \\
\text { programas de formación y las escuelas es garantizar el } \\
\text { apoyo y los recursos suficientes para proporcionar a los futuros } \\
\text { profesores un contexto apropiado donde puedan desarrollar sus } \\
\text { habilidades pedagógicas en TIC y explotarlas más plenamente. }\end{array}$ \\
\hline $\begin{array}{l}\text { Vajravelu } \\
\text { y Muhs } \\
(2016)\end{array}$ & $\begin{array}{l}\text { Estados } \\
\text { Unidos }\end{array}$ & $\begin{array}{l}\text { Integración de tecnología } \\
\text { digital y estrategias } \\
\text { innovadoras para el } \\
\text { aprendizaje y la enseñanza } \\
\text { de clases numerosas: un } \\
\text { estudio de caso de cálculo }\end{array}$ & $\begin{array}{l}\text { Detallaron varios componentes del curso (conjuntos de tareas diarias } \\
\text { en línea, pruebas de habilidades en línea, sesiones de solicitud y } \\
\text { proyectos con Teaching Asistants, pruebas en clase y examen final } \\
\text { completo); discutir cómo obtuvimos resultados óptimos. }\end{array}$ \\
\hline
\end{tabular}

Población impactada

\begin{tabular}{|c|c|c|c|c|}
\hline $\begin{array}{l}\text { Holle- } \\
\text { brands } \\
(2017)\end{array}$ & $\begin{array}{l}\text { Estados } \\
\text { Unidos }\end{array}$ & $\begin{array}{l}\text { Un marco para guiar } \\
\text { el desarrollo de una } \\
\text { enseńanza de matemáticas } \\
\text { con tecnología curso } \\
\text { masivo abierto en línea } \\
\text { para educadores } \\
\text { (MOOC-ED) }\end{array}$ & $\begin{array}{c}\text { Se ofrecen herramientas tecnológicas y sugerencias sobre cómo los } \\
\text { formadores de profesores de matemáticas y los investigadores pueden } \\
\text { ayudarlos a utilizar la tecnología para enseñar esta ciencia. }\end{array}$ & \multirow{4}{*}{$\begin{array}{l}\text { Dirigidos a la } \\
\text { formación del } \\
\text { profesorado de } \\
\text { Matemáticas } \\
\text { en Primaria, } \\
\text { Secundaria } \\
\text { y Universidad }\end{array}$} \\
\hline $\begin{array}{l}\text { Vajravelu } \\
\text { (2018) }\end{array}$ & $\begin{array}{l}\text { Estados } \\
\text { Unidos }\end{array}$ & $\begin{array}{l}\text { Estrategias innovadoras } \\
\text { para el aprendizaje y la } \\
\text { enseñanza de grandes clases } \\
\text { de ecuaciones diferenciales }\end{array}$ & $\begin{array}{c}\text { Señala resultados positivos al desarrollar un método de enseñanza que } \\
\text { incorpora computadoras y software a clases de matemáticas. Como } \\
\text { ejemplo se registra la introducción a la tecnología, la mejora en la } \\
\text { comprensión de los estudiantes. }\end{array}$ & \\
\hline $\begin{array}{c}\text { Gay, } \\
\text { Barry, } \\
\text { Rothrock, } \\
\text { Pelkey } \\
(2020)\end{array}$ & $\begin{array}{l}\text { Estados } \\
\text { Unidos }\end{array}$ & $\begin{array}{l}\text { Opiniones y elecciones } \\
\text { de los profesores de } \\
\text { matemáticas sobre la } \\
\text { enseńanza y los libros } \\
\text { de texto en aulas de } \\
\text { secundaria y preparatoria }\end{array}$ & $\begin{array}{l}\text { Los resultados de la encuesta que aplican sugieren que en la formación } \\
\text { docente los programas están promoviendo las habilidades de } \\
\text { enseñanza recomendadas por las principales autoridades en educación } \\
\text { matemática. Las opiniones de los futuros profesores pueden ser } \\
\text { una guía valiosa para quienes tienen la responsabilidad de tomar } \\
\text { decisiones sobre los libros de texto. Citando el rápido crecimiento en } \\
\text { los libros de texto electrónicos, señalaron la necesidad de realizar más } \\
\text { investigaciones sobre este formato de libro. }\end{array}$ & \\
\hline $\begin{array}{l}\text { Fomina, } \\
\text { Vorobjeva } \\
\text { y Kalitvin } \\
(2016)\end{array}$ & Rusia & $\begin{array}{l}\text { Enfoques de aprendizaje a } \\
\text { distancia en la matemática } \\
\text { Formación de estudiantes } \\
\text { de institutos pedagógicos }\end{array}$ & $\begin{array}{c}\text { La experiencia de la aplicación Moodle en el estudio de la estadística } \\
\text { y la matemática permitió activar el trabajo independiente de los } \\
\text { estudiantes. Los recursos educativos electrónicos son herramientas } \\
\text { auxiliares de formación de los estudiantes que contribuyen a la } \\
\text { profundización de competencias comunicativas, informativas, } \\
\text { cognitivas y permiten generar condiciones especiales para el } \\
\text { autoaprendizaje en los docentes en formación. }\end{array}$ & \\
\hline
\end{tabular}




\begin{tabular}{|c|c|c|c|c|}
\hline Autor & Contexto & Título & Principales aportes & $\begin{array}{l}\text { Población } \\
\text { impactada }\end{array}$ \\
\hline $\begin{array}{c}\text { Moraová } \\
\text { (2017) }\end{array}$ & $\begin{array}{l}\text { República } \\
\text { Checa }\end{array}$ & $\begin{array}{l}\text { ¿Usan los autores de } \\
\text { materiales electrónicos en } \\
\text { línea para la enseñanza de } \\
\text { matemáticas su potencial } \\
\text { para las configuraciones no } \\
\text { estereotípicas culturales? }\end{array}$ & $\begin{array}{l}\text { Los maestros en servicio cuyos materiales fueron analizados en } \\
\text { este estudio no responden a las exigencias del mundo actual. No } \\
\text { aprovechan el potencial del nuevo medio (multimedia en línea, } \\
\text { materiales) y tienden a replicar la misma cultura, patrones con los que } \\
\text { se habían encontrado como estudiantes de primaria y secundaria. }\end{array}$ & \multirow{2}{*}{$\begin{array}{l}\text { Dirigidos a la } \\
\text { formación del } \\
\text { profesorado de } \\
\text { Matemáticas } \\
\text { en Primaria, } \\
\text { Secundaria } \\
\text { y Universidad }\end{array}$} \\
\hline $\begin{array}{l}\text { Brodahl } \\
\text { y Wathne } \\
(2016)\end{array}$ & Noruega & $\begin{array}{l}\text { Percepciones de los } \\
\text { maestros en servicio del } \\
\text { diseńo y de la calidad de } \\
\text { videos de matemáticas en } \\
\text { su aprendizaje en línea }\end{array}$ & $\begin{array}{l}\text { Exploraron autoinformes de los profesores en servicio utilizando } \\
\text { los podcasts. En general, los podcasts parecen generar mayores } \\
\text { habilidades de concentración y, en última instancia, a mejorar la } \\
\text { calidad de enseñanza de las matemáticas. La eficiencia, el disfrute } \\
\text { y la concentración fueron centrales en el profesorado que aprendió } \\
\text { matemáticas a través de podcasts. }\end{array}$ & \\
\hline $\begin{array}{l}\text { Walters, } \\
\text { Green, } \\
\text { Goldsby, } \\
\text { y Parker } \\
\text { (2018) }\end{array}$ & & $\begin{array}{l}\text { La narración digital como } \\
\text { una estrategia de resolución } \\
\text { de problemas en la } \\
\text { formación del profesorado } \\
\text { de matemáticas: cómo } \\
\text { hacer que un matemático } \\
\text { se involucre y emocione a } \\
\text { los estudiantes del } \\
\text { siglo XXI. }\end{array}$ & $\begin{array}{c}\text { Demostraron mediante la aplicación de una encuesta que los maestros } \\
\text { en servicio no estaban familiarizados con los usos digitales en la } \\
\text { enseñanza. No obstante, después de implementar la construcción de } \\
\text { narraciones digitales para la resolución de problemas, la percepción } \\
\text { de estos cambió positivamente. Por tanto, su motivación, disposición } \\
\text { y uso de las tecnologías digitales para la enseñanza de la matemática } \\
\text { aumentó. }\end{array}$ & \multirow{6}{*}{$\begin{array}{c}\text { Estrategias } \\
\text { y/o modelos } \\
\text { de enseńanza } \\
\text { implementados } \\
\text { en el nivel } \\
\text { de Primaria, } \\
\text { Secundaria y } \\
\text { Universidad }\end{array}$} \\
\hline $\begin{array}{l}\text { Maha } \\
(2017)\end{array}$ & $\begin{array}{l}\text { Arabia } \\
\text { Saudita }\end{array}$ & $\begin{array}{l}\text { Uso de Internet en la } \\
\text { enseńanza del álgebra a } \\
\text { estudiantes de secundaria: } \\
\text { un estudio de las } \\
\text { perspectivas y actitudes de } \\
\text { los maestros }\end{array}$ & $\begin{array}{l}\text { Este estudio identificó el grado de conciencia del maestro de } \\
\text { secundaria sobre usar Internet para enseñar y desarrollar el aprendizaje } \\
\text { de álgebra de los estudiantes. Encontró que el Internet en el aula de } \\
\text { álgebra, dentro de este grupo particular de maestros de educación } \\
\text { media, se utiliza para propósitos que no contribuyen a la instrucción } \\
\text { de esta asignatura. }\end{array}$ & \\
\hline $\begin{array}{l}\text { Siaw, } \\
\text { Gyabaah } \\
\text { y Boateng } \\
(2016)\end{array}$ & Ghana & $\begin{array}{l}\text { Los efectos del uso de la } \\
\text { instrucción Microsoft } \\
\text { Math Tool (calculadora } \\
\text { gráfica) en el rendimiento } \\
\text { de los estudiantes en } \\
\text { funciones lineales }\end{array}$ & $\begin{array}{l}\text { El estudio sugirió que los profesores de matemáticas y los } \\
\text { desarrolladores de currículos o libros de texto deberían introducir } \\
\text { el uso de la calculadora gráfica para mejorar el desempeño de los } \\
\text { estudiantes en educación matemática, particularmente para las } \\
\text { funciones lineales y cuadráticas. }\end{array}$ & \\
\hline $\begin{array}{c}\text { Knight, } \\
\text { Casey y } \\
\text { Dekkers } \\
(2017)\end{array}$ & $\begin{array}{l}\text { Estados } \\
\text { Unidos }\end{array}$ & $\begin{array}{l}\text { Uso de libros de texto } \\
\text { electrónicos para enseñar } \\
\text { matemáticas en el aula } \\
\text { secundaria: ¿qué dicen los } \\
\text { estudiantes? }\end{array}$ & $\begin{array}{l}\text { Sus resultados sugieren que el uso de libros de textos electrónicos en } \\
\text { clases de matemáticas en un aula de secundaria tiene efectos positivos } \\
\text { en la percepción de parte de los estudiantes de la utilidad de esta } \\
\text { herramienta y en su motivación. }\end{array}$ & \\
\hline $\begin{array}{l}\text { Yu Yeung } \\
\text { et al. } \\
(2017)\end{array}$ & $\begin{array}{l}\text { Hong } \\
\text { Kong }\end{array}$ & $\begin{array}{c}\text { Explorando las } \\
\text { características de los granos } \\
\text { finos, comportamientos } \\
\text { del aprendizaje de las } \\
\text { matemáticas en actividades } \\
\text { de aprendizaje electrónico } \\
\text { basadas en tabletas }\end{array}$ & $\begin{array}{l}\text { Describieron el progreso en el aprendizaje de las matemáticas en } \\
\text { actividades de e-learning basadas en tabletas. Construyeron una } \\
\text { plataforma experimental, SkyApp, que le permitió a los docentes } \\
\text { realizar el seguimiento de los registros de aprendizaje de los } \\
\text { estudiantes. }\end{array}$ & \\
\hline $\begin{array}{l}\text { Kaur, } \\
\text { Koval y } \\
\text { Chaney } \\
(2017)\end{array}$ & Turquía & $\begin{array}{l}\text { Potencial de usar iPad } \\
\text { como suplemento para } \\
\text { enseñar matemáticas } \\
\text { a estudiantes con } \\
\text { discapacidades de } \\
\text { aprendizaje }\end{array}$ & $\begin{array}{l}\text { Confirmaron el uso del iPad como complemento para ayudar a los } \\
\text { estudiantes en la comprensión de números, orden de operaciones, } \\
\text { expresiones y multiplicación y habilidades de división. }\end{array}$ & \\
\hline
\end{tabular}




\begin{tabular}{llll}
\hline Autor Contexto & Título & Principales aportes & $\begin{array}{c}\text { Población } \\
\text { impactada }\end{array}$ \\
\hline
\end{tabular}

Ferdia
nová $\quad \begin{gathered}\text { República } \\ \text { (2017) }\end{gathered} \quad \begin{gathered}\text { Materiales GeoGebra } \\ \text { para LMS Moodle Monge } \\ \text { enfocado en proyección }\end{gathered}$

El objetivo de este trabajo es introducir materiales interactivos como Geogebra para la proyección de Monge, que se implementan en LMS Moodle, así como mostrar resultados de investigación piloto sobre la influencia del uso de estos materiales en los estudiantes. Señala que GeoGebra resultó como software matemático dinámico para apoyar el aprendizaje de Geometría y sugiere que también puede hacerlo en los campos de la ciencia, la tecnología, la ingeniería (STEM) e innovaciones en la enseńanza y el aprendizaje en todo el mundo.

\begin{tabular}{|c|c|c|c|}
\hline $\begin{array}{l}\text { Dueker; } \\
\text { Cannella- } \\
\text { Malone } \\
(2019)\end{array}$ & $\begin{array}{l}\text { Estados } \\
\text { Unidos }\end{array}$ & $\begin{array}{l}\text { Adición de enseñanza } \\
\text { a los estudiantes con } \\
\text { discapacidades moderadas } \\
\text { mediante la sugerencia } \\
\text { de video }\end{array}$ & $\begin{array}{l}\text { Evidencia el uso de indicaciones de video para enseñar habilidades } \\
\text { (sumas de uno y dos dígitos) a tres estudiantes con discapacidad } \\
\text { moderada. Los resultados indicaron un efectivo progreso de los } \\
\text { estudiantes con relación al manejo de la suma y la aplicación a otros } \\
\text { contextos, participación activa de los participantes y de familiares. }\end{array}$ \\
\hline $\begin{array}{l}\text { Al- } \\
\text { Takhyneh } \\
(2018)\end{array}$ & $\begin{array}{l}\text { Arabia } \\
\text { Saudita }\end{array}$ & $\begin{array}{l}\text { Actitudes hacia el uso } \\
\text { de aplicaciones móviles } \\
\text { en la enseñanza de las } \\
\text { matemáticas en sistemas de } \\
\text { aprendizaje abiertos }\end{array}$ & $\begin{array}{l}\text { Investigó las actitudes hacia la enseñanza de las matemáticas a } \\
\text { través del aprendizaje móvil en sistemas de aprendizaje abiertos. Los } \\
\text { resultados del estudio mostraron que el porcentaje de las actitudes } \\
\text { positivas de los estudiantes hacia el uso de aplicaciones móviles } \\
\text { alcanzó el } 80 \% \text {. }\end{array}$ \\
\hline $\begin{array}{l}\text { Díaz, de } \\
\text { Luna y } \\
\text { Salinas- } \\
\text { Padilla. } \\
\text { (2019) }\end{array}$ & México & $\begin{array}{l}\text { Curso de nivelación } \\
\text { algebraica para incrementar } \\
\text { el rendimiento académico } \\
\text { en estudiantes de ingeniería } \\
\text { en un ambiente virtual de } \\
\text { aprendizaje }\end{array}$ & $\begin{array}{l}\text { Se desarrolló un entorno virtual de aprendizaje tomando como } \\
\text { modelo de diseńo instruccional la estructura Análisis, Diseño, } \\
\text { Desarrollo, Implantación y Evaluación (ADDIE). }\end{array}$ \\
\hline
\end{tabular}

\section{Estrategias y/o modelos de enseñanza implementados en el nivel de Primaria, Secundaria y Universidad}

Proponen un modelo matemático de e-learning MCIEC (motivation, context, interactivity, evaluation, and connectivity) para hacer el aprendizaje matemático más interesante, significativo y aplicable a los alumnos más allá del conocimiento del aula. Registraron hallazgos positivos al implementar una plataforma de aprendizaje electrónico abierto. Alcanzaron altos niveles de interactividad del estudiantado mientras controlaban objetos de aprendizaje. Proponen un modelo matemático de e-learning MCIEC (motivation, context, interactivity, evaluation, and connectivity) para hacer el aprendizaje matemático más interesante, significativo y aplicable a los alumnos más allá del conocimiento del aula. Registraron hallazgos positivos al implementar una plataforma de aprendizaje electrónico abierto. Alcanzaron altos niveles de interactividad del estudiantado mientras controlaban objetos de aprendizaje.

Yong y

zibwe

(2018)
Un modelo de e-learning para la enseńanza de plataforma de aprendizaje de código abierto

para la enseńanza de
Corea $\quad$ las matemáticas en una
plataforma de aprendizaje
de código abierto

Se desarrolló un entorno virtual de aprendizaje tomando como

Universidad

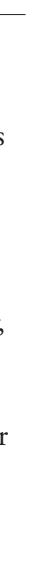

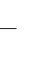


Asimismo, Vajravelu y Mush (2016), y luego Vajravelu (2018), al igual que Ferdinová (2017), reconocen el potencial de las TIC y las herramientas digitales para desarrollar disciplinas STEM e incluso las ciencias sociales.

Como se indicó, ocho de los estudios se desarrollaron en el campo de la formación del profesorado; entre estos destacan Bozkurt, (2016), Brodahl y Wathne (2016), Fomina, Vorobjeva y Kalitvina (2016), Hollebrands (2017), Moraová (2017), Vajravelu (2018), Walters, Green, Goldsby y Parker (2018); o sobre las percepciones o creencias de los docentes relacionadas con aspectos didácticos como Gay, Barry, Rothrock y Pelkey (2020).

Ahora bien, dentro de los hallazgos se pueden citar el potenciar las técnicas tradicionales de enseñanza, tal como lo registra Vajravelu (2018), al incorporar computadores, software y sesiones de aplicación a la resolución de problemas relacionados con ecuaciones diferenciales. Igualmente, Fomina, Vorobjeva y Kalitvina (2016) sostienen que la incorporación de los recursos electrónicos como herramientas auxiliares en la formación del profesorado favorece la profundización de competencias comunicativas, informativas y cognitivas. Asimismo, generan, según lo apuntan los autores citados, condiciones para la mejora de los autoaprendizajes de estudiantes de profesorado.

Brodhal y Wathne (2016) también hallaron que la aplicación de podcasts y los videos promovieron la eficiencia, el disfrute y la concentración como condiciones centrales cuando aprendieron matemáticas a través de estas herramientas tecnológicas.

No obstante, los resultados expuestos por Bozkurt (2016), sobre problemas identificados por los futuros profesores que afectan su uso de la TIC durante las prácticas escolares, tales como: "acceso deficiente a las instalaciones TIC, estímulo limitado por parte de profesores y mentores y uso irregular de las TIC por parte de los actuales profesores formadores, falta de vínculos en el esquema de trabajo y limitación en el tiempo" (p. 91), merecen ser destacados porque dan a conocer los puntos sobre los cuales hay que atender tanto a nivel de políticas públicas, institucional y de formación profesional inicial y continua.
En esa misma línea, Fomina, Vorobjeva y Kalitvina (2016) encontraron que los docentes en servicio de su estudio no parecen aprovechar al máximo el potencial de las TIC y las herramientas multimedia. En contraste, estos, según lo señalan los citados autores, en lugar de responder a los desafíos de la actual sociedad, las condiciones de sus aulas y los intereses de los jóvenes, tienden a replicar los mismos modelos que fueron aplicados cuando eran estudiantes de básica o de secundaria.

Walters, Green, Goldsby y Parker (2018), por su parte, coinciden en los efectos positivos de implementar el uso de herramientas digitales en la enseñanza de las matemáticas por parte del profesorado. En efecto, al implementar una encuesta observaron poca familiaridad del grupo docente con los usos de la tecnología en su práctica de aula. Sin embargo, después de construir narraciones digitales utilizando Math-eos para enseñar conceptos matemáticos y resolver problemas, el profesorado se hizo más consciente de los potenciales usos de las herramientas TIC.

Por otro lado, de las investigaciones que abordaron estrategias de enseñanza en matemáticas mediada por TIC, entornos virtuales o uso de medios digitales para promover aprendizajes significativos en los estudiantes se destacan los artículos de: Díaz, de Luna y Salinas-Padilla (2019), Jeong Yong y Akugizibwe (2018), Knight, Casey y Dekkers (2017), Siaw, Gyabaah y Boateng (2016), Vajravelu y Mush (2016), Vajravelu (2018) y Yu Yeung et al. (2017).

En el caso de Díaz, de Luna y Salinas-Padilla (2019), evidencian que los estudiantes tuvieron con respecto al uso de los entornos virtuales de aprendizaje una apreciación positiva e indicaron que el entorno virtual había apoyado su aprendizaje. Resultados positivos también registraron Jeong Yong y Akugizibwe (2018), al implementar un modelo de aprendizaje e-learning MCIEC (por sus acrónimo en inglés: motivation, context, interactivity, evaluation, and connectivity). Mediante este modelo el aprendizaje de las matemáticas logró ser significativo, interesante y aplicable al contexto e intereses del estudiantado. Las razones por 
las cuales se obtuvieron tales logros es que el modelo enfatiza en la motivación, la contextualización de los aprendizajes a problemas reales y la evaluación dinámica como eje vertebral del diseño y del desarrollo de los contenidos (Jeong y Akugizibwe, 2018).

Por su parte, Knight, Casey y Dekkers (2017) exploraron el uso de libros de texto electrónicos (e-texts) durante las clases de matemáticas en las aulas de secundaria y sugieren también efectos positivos. Se destacan, por ejemplo, un renovado enfoque del aprendizaje y la percepción por parte del estudiantado de ser una herramienta útil y motivante.

Los resultados del estudio cuasi-experimental de Siaw, Gyabaah y Boateng (2016) sugirieron que los profesores de matemáticas deben introducir el uso de la calculadora gráfica para mejorar el desempeño de los estudiantes en esta asignatura.

Igualmente, Vajravelu y Mush (2016) comparten el desarrollo de un método innovador incorporando computadoras y software MyLabsPlus con sesiones de aplicación en clases de cálculo. Sus resultados evidenciaron que la introducción de nuevas tecnologías, la resolución de problemas en clase y las sesiones de aplicación (o discusión) son importantes factores en la mejora de la comprensión profunda de las matemáticas de los estudiantes. Posteriormente, Vajravelu (2018) reitera resultados positivos al implementar una vez más el método de enseñanza que incorporó esta vez computadoras, Canvas (Webcourses@UCF), software WileyPlus y sesiones de aplicación en las clases de matemáticas. Para ilustrar, señala mejora en la comprensión del estudiantado, habilidad para la resolución de problemas y, por supuesto, la introducción a la nueva tecnología.

Del otro lado, Yu Yeung et al. (2017) construyeron una plataforma experimental llamada SkyApp que permitió al profesorado el seguimiento de los registros de los aprendizajes de sus estudiantes y su posterior clasificación, teniendo sus características de aprendizaje de desempeño.

Asimismo, dos investigaciones abordan la enseńanza de la matemática en estudiantes con discapacidades: Dueker y Cannella-Malone (2019) y Kaur, Koval y Chaney (2017). Con relación a los primeros, plantearon el uso de indicaciones de video para la enseñanza de la suma a tres estudiantes con discapacidad moderada. Según lo evidencian los resultados de Dueker y Cannela-Malone (2019), estos afirman que los tres estudiantes mejoraron el manejo de situaciones aditivas inmediatamente después de la instrucción del video. Igualmente, completaron problemas de suma aplicada a otros contextos.

En cuanto a los segundos autores, Kaur, Koval y Chaney (2017), estos muestran cómo el uso de iPads como complemento para enseñar matemáticas a estudiantes con discapacidades pudo ayudarlos a su comprensión de números y encontrar solución a situaciones que requieren la aplicación de operaciones como la multiplicación y la división.

\section{Discusión y conclusiones}

En este aparte del trabajo se apunta a consolidar las respuestas al segundo y tercer objetivo planteados en este estudio: analizar los principales aportes de los artículos revisados en la promoción de una nueva praxis de la enseñanza de las matemáticas y ofrecer recomendaciones pertinentes al profesorado para lograr tal fin.

Inicialmente, se puede acotar que la revisión sobre la enseñanza de las matemáticas mediada por EVA, medios digitales y TIC en publicaciones científicas, divulgadas en la base de datos ERIC y Scielo, permitió obtener información valiosa para el profesorado de matemáticas.

Así, tanto los artículos que abordaron el asunto desde la formación de profesorado inicial y en servicio, como aquellos que lo hicieron desde propuestas o estrategias didácticas implementadas por docentes en servicio para la enseñanza y, por tanto, favorecer el aprendizaje de estudiantes de primaria, secundaria o universitarios, constituyen un provechoso banco de referencias y experiencias dentro del campo de la didáctica de las matemáticas dignas de ser valoradas y evaluadas. Por ejemplo, pudieran transferirse a otros contextos, si es posible, obviamente salvando las distancias. 
En cuanto a los primeros estudios que atienden a la formación inicial o continua del profesorado, se destaca su importancia por cuanto atienden a la cambiante sociedad y a la dificultad de los docentes de enfrentarse a ellas (Rubio y Olivo-Franco, 2020b). Particularmente, las dificultades surgidas a partir de la pandemia del Covid19 prácticamente han obligado a todos los docentes a implementar el trabajo virtual, el uso de las herramientas digitales en el campo de la enseñanza. Aunque precisamente, el impacto de las TIC y este tipo de herramientas digitales es común en la población estudiantil es claro también que su incorporación por parte de los docentes de matemáticas ha sido lenta (Hollebrands, 2017).

Dentro de las dificultades del profesorado más frecuentes señaladas por los artículos revisados pueden citarse: la falta de familiaridad frente a la incorporación de las TIC en la educación matemática o el uso de herramientas digitales (Walters, Green, Goldsby y Parker, 2018); la tendencia a replicar los modelos que fueron aplicados cuando ellos eran estudiantes o transmitir mecánicamente los valores culturales cuando lo que se requiere es comentarlos y trabajar dinámicamente con ellos (Fomina, Vorobjeva y Kalitvin, 2016; Maraova, 2017; Siaw, Gyabaah y Boateng, 2016).

Asimismo, la totalidad de estudios coinciden en reconocer que el profesorado en formación o en servicio reconoce los beneficios del uso de las TIC y los entornos virtuales para la educación matemática. Sin embargo, los estudios evidencian que la mayor parte de los profesores no entienden cuándo y cómo usarlos.

Por consiguiente, es importante poner la atención sobre los anteriores hallazgos dado que el colectivo docente debe tomar conciencia de la importancia de aterrizar su práctica educativa en coherencia con el contexto, los intereses del estudiantado y las dinámicas de las realidades de la sociedad actual; entre estas últimas, el uso necesario de las TIC y las herramientas digitales y su incorporación en el campo de la educación.

Ahora, ¿cómo hacer posible esa incorporación de forma significativa por parte del profesorado de matemáticas? y ¿cómo mejorar la enseñanza y el aprendi- zaje de esta asignatura? Pues bien, con respecto a esta pregunta, los artículos evidencian estrategias variopintas que, a juicio de los procesos validados por la investigación de los autores, han resultado exitosas.

Algunas recomendaciones que pueden develarse al analizar cuidadosamente los trabajos podrían caracterizarse como generales, es decir, independientes a la estrategia, al modelo o a la propuesta que implementaron, pero totalmente necesarias para quienes desean incorporar las TIC a la educación matemática. Entre estas se destacan:

- Trabajar con las TIC y los entornos virtuales demanda incluirlos dentro de un tetraedro didáctico cuyos vértices indican las interacciones entre: estudiante, maestro, el conocimiento de las matemáticas y la mediación de las TIC (Hollebrands, 2017). Exige también del colectivo docente apropiarse del enfoque de aprender a aprender, STEM o gamificación, en los cuales los estudiantes ejercen mayor autorregulación de sus aprendizajes.

- Es necesario utilizar la valiosa información que proveen los contextos de enseñanza y aprendizaje sobre cómo participa el estudiantado a lo largo de las actividades de aprendizaje. El cuidadoso análisis de estas actividades hace posible identificar las necesidades individuales de los estudiantes que requieren especial atención y, posteriormente, diseñar, aplicar y evaluar diferentes acciones pedagógicas que se requieran (Yu Yeung et al., 2017).

- Los roles que puede asumir Internet cuando el profesorado brinda el acceso del estudiantado en el aula pueden ser: 1) Tutor: en caso de que pueden utilizarlo individualmente para estudiar, buscar conceptos matemáticos desconocidos o poco comprensibles; 2) Ayudante: cuando el estudiantado aborde conceptos que no pueden ser considerados sin la ayuda de la tecnología; 3) Entorno: los profesores pueden crear entornos ricos en matemáticas que permitan a los educandos explorar conceptos o interactuar con herramientas virtuales para fortalecer su comprensión de los conceptos matemáticos (Maha, 2017).

- Se recomienda al profesorado reflexionar en torno a interrogantes que no pueden quedar en el aire 
y que orientarán la mediación de las TIC, los EVA o las herramientas digitales y electrónicas que utilice, tales como:

¿Cuál es la demanda cognitiva de la tarea basada en la tecnología que pienso proponer? ¿Cómo interactuará el estudiantado con la tarea y la tecnología? ¿cómo mejora la tecnología o la estrategia propuesta el aprendizaje del estudiante? ¿cómo puedo utilizar la tecnología para facilitar una discusión matemática productiva? ¿Qué tipo de evaluación y de retroalimentación proporciona la tecnología al estudiante? ¿Cómo puedo incorporar la autoevaluación a las tareas? ¿Cómo puedo aprovecharme de la tecnología para determinar lo que están aprendiendo mis estudiantes? (Hollebrands, 2017, p. 86)

Es importante favorecer una pedagogía que "enganche" o involucre al estudiante en un trabajo activo y que incluya el trabajo colaborativo síncrono o asíncrono. Que la retroalimentación sea inmediata es una condición deseable para tener en cuenta al diseñar EVA o propuestas que incorporen las TIC.

Se ofrecen además recomendaciones específicas relacionadas directamente con las propuestas implementadas por los estudios en cuestión y se sugiere sean revisadas detalladamente por el profesorado si se requiere mayor información al respecto; hacerlo en este aparte excede los límites de este trabajo. Ahora bien, lo que sí es posible hacer es explicitar algunas de las herramientas o mediadores TIC utilizados en tales estudios. Entre estos pueden citarse: el uso de libros o narraciones digitales, podcasts, Microsoft Math Tool (calculadoras gráficas), uso de tablas basado en actividades de e-learning, uso de aplicaciones móviles, Geogebra, iPad $y$ videos.

Adicionalmente, los EVA, las TIC y la mediación con herramientas digitales también produjo resultados deseables relacionados con la promoción de estrategias de aprendizaje entre las muestras impactadas, un campo sobre el cual hacen énfasis las investigaciones recientes (Rubio y Olivo-Franco, 2020a). Igualmente, puesto que la mayor parte de los estudios revisados se proponen desde un enfoque constructivista o socioconstructivista, es necesario que los docentes de mate- máticas se familiaricen con los principios teóricos, epistemológicos, ontológicos, axiológicos y filosóficos que lo sustentan.

Por otro lado, en cuanto a los estudios desarrollados como estrategias didácticas y metodológicas para la enseńanza de los contenidos matemáticos y que involucran EVA, TIC o aplicación de herramientas digitales, tales estudios coinciden en que mejoran las técnicas de enseñanza tradicionales y se constituyen en enfoques ingeniosos e innovadores que favorecen la comprensión profunda de las matemáticas por parte de los estudiantes (Olivo-Franco y Corrales, 2020; Siaw, Gyabaah y Boateng 2016; Vajravelu, 2018). Ahora bien, un aspecto que destaca gran parte de las investigaciones es que la incorporación de las tecnologías debe hacer y favorecer la interdisciplinaridad, por tanto, facilitan también trabajar no solo disciplinas del STEM, sino otras como sociales o humanidades (Al-Takhyneh, 2018; Vajravelu, 2018).

Sin embargo, la incorporación de los EVA en la enseńanza de las matemáticas favorece, según la evidencia empírica de los artículos de investigación revisados, no solo el aprendizaje de contenidos matemáticos, sino la habilidad para resolver problemas contextuales, consolida la motivación y el desarrollo de actitudes metacognitivas y autorregulatorias, todas estas competencias muy deseables en la formación del individuo (Bozkurt, 2016, Fomina, Vorobjev y Kalitvin, 2016; Jeong Yong y Akugizibwe, 2018; Olivo 2017; Olivo-Franco, 2019; y Olivo-Franco y Corrales, 2020). Interesantemente, los resultados positivos se han logrado no solo con muestras de profesores de matemáticas de primaria, secundaria y universitarios, o con estudiantes de estos niveles educativos, sino también con estudiantes con discapacidades o con trastornos emocionales (Kaur, Koval y Chaney, 2017; Zhang, Trusell, Gallegos, Asam, 2015).

En este último grupo de estudiantes las estrategias de incorporación de las TIC privilegiaron aspectos como la motivación, la promoción de la independencia, la contextualización, el juego, la gamificación y el incentivar el trabajo en equipo. Tales aspectos han de tenerse en cuenta también a la hora de pensar en los EVA. 
No obstante, tener disponibles hoy más que nunca los EVA y las TIC no garantiza per se que se resignifique la praxis educativa de los docentes de matemáticas y facilitar el aprendizaje matemático para los estudiantes. Pues precisamente con tantas herramientas digitales los docentes pueden fácilmente convertirse en copiadores en lugar de creadores de tales recursos. Por consiguiente, uno de los desafíos es poder mantener el equilibrio y el cuidado en el uso de la tecnología, seleccionando con asertividad el material necesario que permita el logro de los objetivos de aprendizaje de acuerdo a las necesidades de cada estudiante (Hollibrands, 2017).

No perder de vista que la enseñanza y el aprendizaje son procesos humanos cargados profundamente de emociones debe también tenerse en cuenta a la hora de integrar o crear entornos virtuales de aprendizaje desde la enseñanza. En este punto es necesario atender a los hallazgos en el área de la neuroeducación y, por ejemplo, las pautas de Cowan y Morey (2007), que indican que las personas aprenden más cuando hay palabras e imágenes y, en el caso de los mensajes multimedia, cuando su diseño atiende consistentemente cómo funciona la mente humana.

Como limitaciones del presente estudio se debe reconocer que es una sencilla revisión documental o bibliográfica concebida, tal ya se ha dicho, como investigación. Esto significa que los hallazgos, en su mayoría, fueron descriptivos en tanto que desarrollan una caracterización de los artículos seleccionados. Si bien se abordan características en las cuales convergen estos, como por ejemplo, metodología empleada, contexto y población para la cual fueron dirigidos, se podría desarrollar una revisión sistemática propiamente dicha. Hacerlo permitiría aguzar o profundizar en el análisis de los datos de forma más potente $y$, por consiguiente, generar conclusiones de mayor alcance. No obstante, puede pensarse en lo anterior como prospectiva precisamente de esta investigación.

Finalmente, un desafío enorme en el contexto latinoamericano en cuanto a la integración de EVA, TIC y herramientas digitales sigue siendo la enorme brecha de desigualdad social de la gran parte de países que la conforman (Olivo-Franco, 2019b). Esto incluye, entre otros aspectos, el acceso a este tipo de recursos, la conectividad de muchas regiones, particularmente en el contexto colombiano, que han quedado evidenciadas notoriamente en los actuales momentos de pandemia. Tales desafíos por parte de los estados deberían subsanarse y asegurarse que todos y todas, sin importar el estrato o la condición, accedieran con facilidad a este tipo de entornos.

\section{Referencias}

Al-Takhyneh, B. (2018). Attitudes towards Using Mobile Applications in Teaching Mathematics in Open Learning Systems. International Journal of E-learning \& Distance Education, 33(1), 1-16. https://files.eric.ed.gov/fulltext/EJ1 180063.pdf

Bertanlanffy, L. (1974). Robots, hombres y mente: la psicología en el mundo moderno. Guadarrama.

Bozkurt, G. (2016). Mathematics teachers and ICT: Factors affecting pre-service use in school placements. International Journal of Research in Education and Science (IJRES), 2(2), 453-468. https://files.eric.ed.gov/fulltext/EJ1110260.pdf

Brodahl, C. \& Wathne, U. (2016). In-service teachers' perceptions of the design and quality of mathematics videos in their on-line learning. Journal of the International Society for Teacher Training, 20(2), 67-78. https://eric.ed.gov/?id= EJ1177048

Cowan, N. \& Morey, C. C. (2007). How can dual-task working memory retention limits be investigated? Psychological Science, 18(8), 686-688. https://doi. org/10.1111/j.1467-9280.2007.01960.x

Day, R.A. (2005). Cómo escribir y publicar trabajos cientificos ( $3^{\mathrm{a}}$ ed.). Organización Panamericana de Salud.

Díaz, J., Lagunes, C., López, M. y Recio, C. (2012). El video como auxiliar didáctico en el rendimiento académico de matemáticas a nivel superior. En Flores, R. (Ed.), Acta Latinoamericana de Matemática Educativa (pp. 265-274). Comité Latinoamericano de Matemática Educativa. http://funes.uniandes.edu.co/4158/1/DiazElvideoALME2012.pdf. 
Díaz, J., de Luna, M. y Salinas-Padilla, H. (2019). Curso de nivelación algebraica para incrementar el rendimiento académico en estudiantes de ingeniería en un ambiente virtual de aprendizaje. Revista Iberoamericana para la Investigación y el Desarrollo Educativo, 9(18), 456-489. https:// doi.org/ 10.23913/ride.v9i18.432

Driscoll, M. (2000). Psychology of Learning for Instruction. Allyn \& Bacon.

Dueker, S. \& Canella-Malone, H. (2019). Teaching Addition to Students with Moderate Disabilities Using Video Prompting. The Journal of Special Education Apprenticeship, 8(2), 1-20. https://files. eric.ed.gov/fulltext/EJ1231811.pdf

Ferdianová, V. (2017). GeoGebra Materials for LMS Moodle Focused Monge on Projection. The Electronic Journal of e-Learning, 15(3), 259-268. https://eric.ed.gov/?id=EJ1146049

Fomina, T., Vorobjev, G., \& Kalitvin, V. (2016). Distance Learning Approaches in the Mathematical Training of Pedagogical Institutes's Students. International Journal of Environmental \& Science Education, 11(18), 12145-12154. https://eric.ed. gov/?id=EJ1122664

Gay, A.S., Barry, A.L., Rothrock, K.S., \& Pelkey, M.M. (2020). Mathematics student teachers' views and choices about teaching and textbooks in middle and high school classrooms. International Journal of Research in Education and Science (IJRES), 6(1), 120-132. https://files. eric.ed.gov/fulltext/EJ1229007.pdf

Gómez, O. A. (2011). Ruta de apoyo pedagógico para la enseñanza de geometría y trigonometría, en el curso 'matemáticas básicas' de la Universidad Nacional de Colombia Sede Medellin. [Tesis doctoral, Universidad Nacional de Colombia].

González, N. E. (2013). Factores asociados a una evaluación académica en la enseñanza de matemática: herramienta estratégica para incrementar la calidad de la enseñanza y el aprendizaje: Acta Latinoamericana de Matemática Educativa (pp. 899-906). México, DF: Comité Latinoamericano de Matemática Educativa. http://funes.uniandes.edu. co/4176/
Guirao-Goris, J.A., Olmedo, A. y Ferrer, E. (2008). El artículo de revisión. Revista Iberoamericana De Enfermeria Comunitaria, 1(1), 1-25. https:// doi.org/10.15665/rp.v15i1.679.s191

Hernández, R., Fernández, C. y Baptista. (2010). Metodología de la investigación (5 a ed.) McGrawHill/Interamericana.

Hollebrands, K. (2017). A framework to guide the development of a teaching mathematics with technology massive open online course for educators (MOOC-ED). En Galindo, E., y Newton, J., (Eds.) (pp. 80-89). Proceedings of the 39th annual meeting of the North American Chapter of the International Group for the Psychology of Mathematics Education. Hoosier Association of Mathematics Teacher Educators.

Jeong Yong A. \& Akugizibwe E. (2018). An e-Learning Model for Teaching Mathematics on an Open Source Learning Platform. International Review of Research in Open and Distributed Learning, 19(5), 255-267. https://eric.ed.gov/?id= EJ1198098

Kaur, D., Koval, A. \& Chaney H. (2017). Potential of using iPads as a supplement to teach math to students with learning disabilities. International Journal of Research in Education and Science (IJRES), 3(1), 114-121. https://files.eric.ed.gov/ fulltext/EJ1126733.pdf

Knight, B., Casey, M., y Dekkers, J. (2107). Using electronic textbooks to teach mathematics in the secondary classroom: What do the students say? International Journal of Education and Development using Information and Communication Technology (IJEDICT), 13(1), 87-102. http:// ijedict.dec.uwi.edu/viewarticle.php?id=2210.

Maha, S. (2017). Using The Internet In Teaching Algebra To Middle School Students: A Study Of Teacher Perspectives And Attitudes. Contemporary Issues in Education Research-Second Quarter, 10(2), 121-136. https://files.eric.ed.gov/fulltext/ EJ1137700.pdf

Maturana, H. y Varela, F. (1990). El árbol del conocimiento. Las bases biológicas del conocimiento. Debate. 
Martínez, M. (2012). Comportamiento Humano. Nuevos métodos de investigación. Trillas.

Moraová H. (2017). "Do Authors of Online Electronic Materials for Teaching Mathematics use Their Potential to use Non-Stereotypical Cultural Settings?" The Electronic Journal of e-Learning, 15(3), 235-243. https://files.eric.ed.gov/fulltext/EJ1146046.pdf

Morales, O. (2015). Fundamentos de la investigación documental y la monografía. En Introducción a los métodos y técnicas de investigación. Selección de lecturas, 213-212. Editorial Universitaria Félix Varela.

Moreno, J. (2003). Tres teorías que dieron origen al pensamiento complejo: Sistémica, Cibernética e Información. En Manual de iniciación pedagógica al pensamiento complejo, 38-58. UNESCO.

Morin, E. (1996). Introducción al pensamiento complejo. Gedisa.

Olivo-Franco, J. (2017). Caracterización de estudiantes exitosos: Una aproximación al aprendizaje de las Ciencias Naturales. CPU-e. Revista de Investigación Educativa, 25, 114-143. http:// www.scielo.org.mx/pdf/cpue/n25/1870-5308cpue-25-00114.pdf

Olivo-Franco, J. (2019a). Interpretativa de docentes sobre estudiantes exitosos. Revista Complutense de Educación, 30(2), 17-34. https://dx.doi.org/ 10.5209/RCED. 57395

Olivo Franco, J. (2019b). Dewey, Freire y Maturana: educación y democracia una deuda pendiente en Latinoamérica. DELECTUS -Revista cientifica, INICC-PERÚ, 2(1), 9-28. https://revista. inicc-peru.edu.pe/index.php/delectus/article/ view/4

Olivo-Franco, J. y Corrales, J. (2020). De los entornos virtuales de aprendizaje: hacia una nueva praxis en la enseñanza de la matemática. Revista Andina De Educación, 3(1), 8-19. https://doi.org/10.32 719/26312816.2020.3.1.2

Organización de las Naciones Unidas para la Educación, la Ciencia y la Cultura-Laboratorio Latinoamericano para la Educación en América Latina y el Caribe ([UNESCO/LLECE]. (2015).
Informe de los Resultados del Tercer Estudio Regional Comparativo: Logros de aprendizaje [TERCE]. https://unesdoc.unesco.org/ark:/ 48223/pf0000243532

Organización de las Naciones Unidas para la Educación, la Ciencia y la Cultura/ Oficina Regional de Educación para América Latina y el Caribe [UNESCO/OREAL]. (2016). Educación para transformar vidas. Metas opciones de estrategia $e$ indicadores. Agenda E 2030. http://www. unesco.org/new/es/santiago/education-2030/ e2030-documents/

Orozco-Alvarado, J. y Díaz-Pérez, A. (2018). ¿Cómo redactar los antecedentes en una investigación cualitativa? Revista Electrónica de Conocimientos, Saberes y Prácticas, 1(2), 66-82. https://doi. org/10.30698/recsp.v1i2.13

Osorio, S. (2012). El pensamiento complejo y la transdisciplinariedad: fenómenos emergentes de la nueva racionalidad. Revista de la Facultad de Ciencias Económicas: Investigación y Reflexión, 20(1), 269-291.

Perales, F. J. (2018). El área de Didáctica de las ciencias Experimentales en España: entre la tribulación y la esperanza. Ápice. Revista de Educación Científica, 2(2), 1-14. https://doi.org/10.17979/ arec.2018.2.2.3915

Petriz, M., Barona, C., López R. y Quiroz J. (2010). Niveles de desempeño y actitudes hacia las matemáticas en estudiantes de la licenciatura en administración en una universidad estatal mexicana. Revista mexicana de investigación educativa, 15(47), 1223-1249. http://www. scielo.org.mx/scielo.php?script=sci_arttext $\&$ pid $=S 1405-66662010000400012$

Rubio, F. y Olivo-Franco, J. (2020a). Revisión de los programas de enseñanza de estrategias de aprendizaje evaluados en Espańa. Ikastorratza. e-Revista de Didáctica, 24, 16-40. https://doi. org/10.37261/24_alea/2

Rubio, F., y Olivo-Franco, J. (2020b). Dificultades del profesorado en sus funciones docentes y posibles soluciones. Un estudio descriptivo actualizado. Ciencia y Educación, 4(2), 7-25. https:// doi.org/10.22206/cyed.2020.v4i2.pp7-25 
Seitzinger, J. (2006). Be Constructive: Blogs, Podcasts, and Wikis as Constructivist Learning Tools. Learning Solutions e-Magazine, July 31, 1-12.

Siemens, G. (2004). Connectivism: A Learning Theory for the Digital Age. International Journal of Instructional Technology and Distance Learning, 2.

Siaw, P. Gyabaah. \& Boateng, S. (2016). The Effects of the use of Microsoft Math Tool (Graphical Calculator) instruction on students' performance in linear functions. Journal of Education and Practice, 7(21), 117-127. ISSN 2222-288X (Online). https://eric.ed.gov/?id=EJ1109378

Solórzano, F. y García, A. (2016). Fundamentos del aprendizaje en red desde el conectivismo y la teoría de la actividad. Revista cubana de Educación Superior, 35(3), 98-112. http://scielo.sld. $\mathrm{cu} /$ scielo.php?script=sci_arttext\&pid=S025743142016000300008

Stošic, L. (2015). The importance of educational technology in teaching. International Journal of Cognitive Research in Science, Engineering and Education (IJCRSEE), 3(1), 111-114. http:// oaji.net/articles/2015/1014-1434799607.pdf

Vargas, G. (1988). Algunas características epistemológicas de la investigación documental. Ascolbi, $1(3)$.

Vajravelu, K. \& Muhs, T. (2016). Integration of digital technology and innovative strategies for learning and teaching large classes: A calculus case study. International Journal of Research In Education and Science (IJRES), 2(2), 379-395. https:// files.eric.ed.gov/fulltext/EJ1105125.pdf
Vajravelu, K. (2018). Innovative Strategies for Learning and Teaching of Large Differential Equations Classes. International Electronic Journal of Mathematics Education. E-ISSN: 1306-3030, 13(2), 91-95. https://doi.org/10.12973/iejme/2699

Vélez, O, y Galeano, M. (2002). Investigación cualitativa Estado del arte. Universidad de Antioquia. Facultad de Ciencias Sociales y Humanas. Centro de Investigaciones Sociales y Humanas CISH.

Vygotsky, L. S. (1968). Pensamiento y Lenguaje. Editorial Pueblo y Educación.

Walters, L.M., Green, M.R., Goldsby, D., \& Parker, D. (2018). Digital storytelling as a problem-solving strategy in mathematics teacher education: How making a math-eo engages and excites 21 st century students. International Journal of Technology in Education and Science (IJTES), 2(1), 1-16. https://files.eric.ed.gov/fulltext/EJ1 227054.pdf

Yu Yeung, C., Hong Shum, K., Chi Kwong Hui, L., Wah Chu, K., Yun Chan, T., Nin Kuo, Y., \& Ling Ng, Y. (2017). Exploring characteristics of fine-grained behaviors of learning mathematics in tablet-based e-learning activities. International Conference e-Learning 2017, 149-153. https:// files.eric.ed.gov/fulltext/ED579335.pdf

Zhang, M., Trussell, R. P., Gallegos, B., \& Asam, R. R. (2015). Using math apps for improving student learning: An exploratory study in an inclusive fourth grade classroom. Techtrends. Linking Research And Practice To Improve Learning, 59(2), 32-39. 